\title{
Dalbavancin for the treatment of acute bacterial skin and skin structure infections
}

\author{
Sheena Ramdeen, MD ${ }^{1}$ and Helen W Boucher, MD FIDSA FACP ${ }^{\dagger, 2}$ \\ ${ }^{1}$ Tufts University School of Medicine, Tufts Medical Center, Division of Geographic Medicine and \\ Infectious Diseases, Boston, MA, USA \\ 2Director Infectious Diseases Fellowship Program, Associate Professor of Medicine, Tufts \\ University School of Medicine, Tufts Medical Center, Division of Geographic Medicine and \\ Infectious Diseases, Boston, MA, USA
}

\section{Abstract}

Introduction-Acute bacterial skin and skin structure infections (ABSSSI) have increased in incidence and severity. The involvement of resistant organisms, particularly methicillin-resistant Staphylococcus aureus, presents additional challenges. The lipoglycopeptide dalbavancin has a prolonged half-life, high protein binding, and excellent tissue levels which led to its development as a once-weekly treatment for ABSSSI. In the pivotal DISCOVER 1 and DISCOVER 2 trials, dalbavancin proved non-inferior to vancomycin followed by linezolid when used sequentially for ABSSSI, forming the basis for its recent approval in the US and Europe for ABSSSI.

\begin{abstract}
Areas covered-A literature search of published pharmacologic and clinical data was conducted to review the chemistry, pharmacodynamics, and pharmacokinetics of dalbavancin. We also discuss its development process, highlighting efficacy and safety data from pertinent clinical trials and the role it could play in the current clinical landscape.
\end{abstract}

Expert opinion-DISCOVER 1 and DISCOVER 2 demonstrated dalbavancin's non-inferiority to vancomycin followed by linezolid for ABSSSI and confirmed its safety and tolerability. They were among the first trials to use new, early primary efficacy endpoints, and dalbavancin was among the first agents designated a Qualified Infectious Disease Product for expedited review. Dalbavancin may prove to be a valuable option for ABSSSI patients in whom conventional therapy is limited.

\section{Keywords}

acute bacterial skin and skin structure infections; dalbavancin; gram-positive bacteria; lipoglycopeptide

\footnotetext{
${ }^{\dagger}$ Author for correspondence: Tel: +1 617636 3010; Fax: +1 617636 8525; hboucher@tuftsmedicalcenter.org. Declaration of interest

The authors have no other relevant affiliations or financial involvement with any organization or entity with a financial interest in or financial conflict with the subject matter or materials discussed in the manuscript apart from those disclosed.
} 


\section{Introduction}

Acute bacterial skin and skin structure infections (ABSSSI) was recently defined by the FDA as a bacterial infection of the skin with a lesion size area of at least $75 \mathrm{~cm}^{2}$, measured by redness, edema, or induration [1]. ABSSSI represent a significant burden on the healthcare system, with increasing incidence and severity in recent years. Admissions for ABSSSI in the United States exceed 860,000 annually, a 29\% increase from 2000 to 2004 [2]. Annual outpatient visits for ABSSSI nearly doubled from 1997 to 2005 [3]. Between 2006 and 2010, about 34.8 million ABSSSI cases were seen in ambulatory settings, 33\% in the Emergency Department [4]. Yet these figures may underestimate the true magnitude of ABSSSI; approximately $50 \%$ of people with moderately severe infections reported they would self-treat without medical care [5].

The most notable factor in the rise of ABSSSI may be the emergence of communityacquired methicillin-resistant Staphylococcus aureus (MRSA) [6]. From 2001 to 2009, hospitalizations for $S$. aureus ABSSSI rose from 160,811 to 358,212, a $123 \%$ increase, and S. aureus ABSSSI incidence doubled [7]. S. aureus is by far the most commonly isolated pathogen in cultures of ABSSSI, and MRSA accounts for nearly half of these isolates [8]. The empirical use of antibiotics for MRSA has mirrored the upward trajectory of ABSSSI in the past decade [3]. Though MRSA is less prevalent in Europe than in the United States, it poses a similar public health challenge $[9,10]$.

Infectious Diseases Society of America guidelines recommend therapy with a $\beta$-lactam or clindamycin for mild/moderate, non-purulent ABSSSI and vancomycin plus piperacillin/ tazobactam for severe, non-purulent ABSSSI [11]. Treatment of purulent ABSSSI should cover MRSA empirically with doxycycline or trimethoprim/sulfamethoxazole (TMP/SMX) in moderate cases and vancomycin, daptomycin, linezolid, televancin, or ceftaroline in severe cases [11]. With increasing clinical MRSA isolates with decreased susceptibility or resistance to these drugs, treatment of ABSSSI is now challenged by antibiotic resistance, toxicity, few oral options, and greater need for hospitalization and its associated costs [12-14].

Dalbavancin (Box 1), a new addition to the antimicrobial armamentarium that could meet these challenges, is a novel lipoglycopeptide approved by the FDA in May 2014 and by the European Medicines Agency (EMA) in February 2015 for ABSSSI caused by susceptible Gram-positive organisms $[15,16]$. This review will discuss its pharmacologic properties, data from pertinent clinical trials, and the role it could play in the current clinical landscape.

Data and other information for this review were obtained from a PubMed/MEDLINE electronic database search for English language articles from February 2002 through April 2015 that contained keywords dalbavancin, lipoglycopeptide, and skin and soft tissue infections.

\section{Overview of the market}

Antimicrobials available in the US for treating ABSSSI with activity against MRSA and other resistant Gram-positive pathogens include vancomycin, daptomycin, linezolid, 
televancin, ceftaroline, dalbavancin, oritavancin, and tedizolid. Oritavancin and tedizolid proved non-inferior to vancomycin and linezolid, respectively, for ABSSSI and were approved by the FDA in 2014 [17,18]. Of the antimicrobials listed, only linezolid and tedizolid have oral formulations, and some have significant potential for toxicity, including renal impairment from vancomycin, bone marrow suppression, and drug interactions (e.g., selective serotonin re-uptake inhibitors) from linezolid, and myopathy from daptomycin $[14,19]$. While doxycycline and TMP/SMX have MRSA activity, their activity against $\beta$ hemolytic streptococci is poorly understood, limiting their use as monotherapy for ABSSSI [11].

There are several compounds in development that may have future indications for treating ABSSSI. These include the quinolones delafloxacin, avarofloxacin, finafloxacin, and nemonoxacin; the tetracycline omadacycline; the oxazolidinones radezolid and MRX-I; the defensin-mimetic Brilacidin; the pleuromutilin lefamulin; the glycopeptide-cephalosporin heterodimers TD-1702 and TD-1607; the FabI inhibitors CG-400549 and Debio 1452, as well as its pro-drug Debio 1450, and the type 2 topoisomerase inhibitor GSK2140944 $[20,21]$.

\section{Introduction to the compound}

Dalbavancin is a semi-synthetic lipoglycopeptide that, like other glycopeptides, binds to the terminal $\mathrm{D}$-alanyl-D-alanine peptidoglycan sequence of the Gram-positive bacterial cell wall. This forms a complex that prevents transpeptidation and subsequent transglycosylation, interfering with cross-linking and polymerization in the bacterial cell wall for a bactericidal effect [22]. As a lipoglycopeptide, dalbavancin is characterized by a long, lipophilic side chain. This side chain allows dalbavancin to dimerize and anchor in the bacterial cell membrane, increasing stabilization and interaction with bacterial peptidoglycans [22]. The $\mathrm{N}$-acylglucosamine moiety results in a long plasma terminal half-life that makes it unique among drugs used to treat ABSSSI [22].

\subsection{Chemistry}

Dalbavancin is derived from the naturally occurring glycopeptide A 40926, which was isolated from Nonomuria spp. through a three-step procedure involving selective esterification of the $\mathrm{N}$-acyclaminoglucuronic acid function, amidation of the peptidecarboxy group, and saponification of the sugar methyl ester [22]. Neither this process nor additional minor modifications affected the $\mathrm{D}$-alanyl-D-alanine binding pocket [22].

Determination of the crystal structure of dalbavancin identified molecular features that likely enhance its antimicrobial activity [23]. Dalbavancin's structure is more closed than its parent compound, and the dimerization that occurs between dalbavancin pairs at the ${ }_{\mathrm{D}}$-alanyl-Dalanine target site is a loose association unlike the hydrogen-bonded dimers noted with other glycopeptide antimicrobials [23]. Dalbavancin's C-terminal dimethyl-propylamine group is free and flexible, supporting the hypothesis that it enhances activity by inserting into the bacterial membrane [23]. The fatty acyl group allows nonspecific protein binding that likely contributes to dalbavancin's long plasma half-life and its ability to bind its target sites [23]. 


\subsection{Pharmacodynamics}

Dalbavancin is highly protein-bound (93\%), particularly to albumin, leading to a steadystate volume of distribution $\approx 10-121$ and a prolonged half-life $\approx 240 \mathrm{~h}$; the initial free drug concentration after a $1 \mathrm{~g}$ dose is approximately $21 \mathrm{mg} / 1$ [24]. An in vitro pharmacokinetic model using four $S$. aureus strains with decreasing dalbavancin concentrations to simulate a $240 \mathrm{~h}$ half-life noted concentration-independent killing with initial free drug concentrations of $3-21 \mathrm{mg} / 1$ [24]. Another in vitro study found that dalbavancin exhibited time-dependent killing against 146 staphylococci strains and was more potent than other agents [25]. An evaluation of both time-dependent and concentration-dependent pharmacodynamics of dalbavancin against $S$. aureus and Streptococcus spp. found that estimated susceptibility breakpoints using both $\mathrm{AUC}_{14 \text { days }} / \mathrm{MIC}$ and $t>\mathrm{MIC}$ for all isolates were well above the known dalbavancin $\mathrm{MIC}_{90} \mathrm{~s}$, further supporting the use of once-weekly dosing of dalbavancin [26].

The rat granuloma pouch model demonstrated greater efficacy of dalbavancin versus vancomycin and linezolid against $S$. aureus, with $>2 \log \mathrm{CFU} / \mathrm{ml}$ reduction and prevention of regrowth at $120 \mathrm{~h}$ with a single $10 \mathrm{mg} / \mathrm{kg}$ intravenous dose [27]. The pharmacodynamics of dalbavancin in neutropenic murine thigh and lung models demonstrated dose-dependent bactericidal activity, which correlated well with in vivo dalbavancin activity, supporting the use of large, infrequent doses [28].

Multiple studies have established dalbavancin's potent in vitro activity against a variety of Gram-positive isolates from both community and hospital settings [29-42]. S. aureus $\mathrm{MIC}_{90} \mathrm{~s}$ are typically $0.06 \mu \mathrm{g} / \mathrm{ml}$, ranging from $0.06-0.25 \mu \mathrm{g} / \mathrm{ml}$, and $\beta$-hemolytic streptococci $\mathrm{MIC}_{90} \mathrm{~s}$ range $0.03-0.25 \mu \mathrm{g} / \mathrm{ml}$ [29-41]. Dalbavancin is also active against coagulase-negative staphylococci, viridans group streptococci, enterococci (excluding VanA phenotype), Corynebacterium spp., Bacillus spp. Listeria spp., and several anaerobes $[29-31,34,42,43]$.

Dalbavancin demonstrated superior in vitro activity versus comparators, including vancomycin, with dramatically lower MICs without evidence of resistance through direct selection or serial passage [29-37,43]. Dalbavancin has maintained this in vitro potency for over a decade without evidence of emergent resistance [38-41]. There has been no evidence to date to suggest that its long half-life would contribute to the development of resistance.

A synergy study of dalbavancin and nine antimicrobial classes did not show any antagonism [44]. The study noted synergy or partial synergy between dalbavancin and oxacillin for staphylococci, including MRSA and VISA, and enterococci [44]. Further investigation is necessary to determine the clinical utility of this synergistic effect.

\subsection{Pharmacokinetics and metabolism}

Like other glycopeptides, dalbavancin has poor oral absorption and requires intravenous administration, but its high protein binding and long half-life make it unique in its class [24]. Pharmacokinetic studies in a rat model noted a plasma concentration-time profile consistent with a three-compartment model, wide tissue distribution, a terminal half-life of $124-188$ $\mathrm{h}$, and excretion via both renal and non-renal routes [45]. 
A Phase I double-blind, randomized, placebo-controlled, single- and multiple-dose study had similar results [46]. Those in the single-dose group received 140, 350, 500, 630, 840, or $1120 \mathrm{mg}$ of dalbavancin. The multiple-dose groups received a loading dose of 300, 400, 600,800 , or $1000 \mathrm{mg}$ divided into two equal doses administered $12 \mathrm{~h}$ apart followed by six daily doses of 30, 40, 60, 80, and $100 \mathrm{mg}$, respectively. Here dalbavancin exhibited doseproportional, linear pharmacokinetics, a steady state volume of distribution of approximately $10 \mathrm{l}$, a terminal half-life between 149 and $198 \mathrm{~h}$, and 33.5\% renal excretion [46]. Blister fluid concentration was concordant with plasma concentration, and serum bactericidal activity at plasma concentrations $\geq 20 \mathrm{mg} / \mathrm{l}$, attainable with once weekly dosing, support the use of dalbavancin for ABSSSI $[46,47]$. Another study confirmed that dalbavancin concentrations in blister fluid over 7 days remained above the $\mathrm{MIC}_{90}$ values for typical ABSSSI pathogens [48]. A recent Phase I study measured total dalbavancin concentrations in plasma, bone, articular tissue, and surrounding skin at 12, 24, 72, 168, 240, and $336 \mathrm{~h}$ after a single $1000 \mathrm{mg}$ dose [49]. The mean concentrations in skin at all time points were well above the $\mathrm{MIC}_{90}$ values for common ABSSSI pathogens.

A population pharmacokinetics study of dalbavancin $1000 \mathrm{mg}$ intravenously on day 1 and $500 \mathrm{mg}$ on day 8 demonstrated a two-compartment model with first-order elimination [50]. Body surface area (BSA) predicted central volume of distribution, and both BSA and creatinine clearance $\left(\mathrm{CL}_{\mathrm{CR}}\right)$ predicted dalbavancin clearance [50]. Dalbavancin clearance was not affected by age, gender, race, serum albumin, cytochrome P450 substrates, inducers, or inhibitors, or concomitant medications [50]. Overall, pharmacokinetic studies suggest low inter-individual variability, and subtherapeutic levels have not been observed.

Dalbavancin pharmacokinetics was examined in patients with varying degrees of hepatic and renal impairment. When administered as a single dose (500 or $1000 \mathrm{mg}$ ) or two doses (1000 mg followed by $500 \mathrm{mg} 1$ week later), only severe renal impairment $\left(\mathrm{CL}_{\mathrm{CR}}<30 \mathrm{ml} /\right.$ min) showed a significant increase in mean $\mathrm{AUC}_{0-\infty}$ [51]. These patients should be treated with $750 \mathrm{mg}$ followed by $375 \mathrm{mg} 1$ week later; no dose adjustment is necessary for $\mathrm{CL}_{\mathrm{CR}} \geq$ $30 \mathrm{ml} / \mathrm{min}$, hemodialysis, or any degree of hepatic impairment [51]. Of note, further studies are needed to evaluate the pharmacokinetics of dalbavancin in critically ill patients since low albumin levels and increased volume of distribution may affect serum levels and clinical efficacy.

\section{Clinical efficacy}

A randomized, controlled, open-label Phase II proof-of-concept trial compared dalbavancin to standard agents for treating ABSSSI in 62 adults from 2001 to 2002 [52]. Subjects received either $1100 \mathrm{mg}$ of dalbavancin as a single infusion $(\mathrm{n}=20), 1000 \mathrm{mg}$ of dalbavancin intravenously followed by $500 \mathrm{mg}$ intravenously 1 week later $(\mathrm{n}=21)$, or a comparator (ceftriaxone, cefazolin, piperacillin/tazobactam, clindamycin, vancomycin, linezolid, or cephalexin, used individually or in combination, $\mathrm{n}=21$ ) for 7 to 21 days. Clinical and microbiological success rates were similar among groups, with a trend to favor the two-dose dalbavancin regimen. There were no differences in drug-related adverse events among the groups, and all regimens were well tolerated [52]. 
An international, multicenter, Phase III, non-inferiority study from 2003 to 2004 randomized well-matched patients with complicated ABSSSI known or suspected to involve MRSA in a 2:1, double-blind manner to receive a two-dose dalbavancin regimen $(1000 \mathrm{mg}$ intravenously on day 1 and $500 \mathrm{mg}$ intravenously on day $8, \mathrm{n}=571$ ) or linezolid (600 mg intravenously or orally every $12 \mathrm{~h}$ for 14 days, $\mathrm{n}=283$ ) [53]. Clinical success rates were $\geq$ $90 \%$, and a microbiological response was $>85 \%$ in evaluable patients in both arms. Adverse events were mild and slightly higher in the linezolid arm (32.2 vs $25.4 \%$ ).

DISCOVER 1 and DISCOVER 2, identically designed, Phase III, international, multicenter, randomized, double-blind, double-dummy trials demonstrated the non-inferiority of dalbavancin versus vancomycin followed by linezolid when used in a sequential manner for treatment of ABSSSI (Table 1) [54]. From 2011 to 2012, well-matched patients, many of whom were seriously ill, received either the previously described two-dose regimen of dalbavancin ( $\mathrm{n}=659$ ) or vancomycin at a dose of $1 \mathrm{~g}$ (or $15 \mathrm{mg} / \mathrm{kg}$ of body weight) intravenously every $12 \mathrm{~h}$ for at least 3 days with the option to switch to linezolid $600 \mathrm{mg}$ orally every $12 \mathrm{~h}$ to complete 10 to 14 days of therapy $(\mathrm{n}=653)$ [54]. DISCOVER 1 included slightly more patients with major abscesses, while DISCOVER 2 included more with cellulitis, but the infected area in both far exceeded the minimum size of $75 \mathrm{~cm}^{2}$ with median values $>300 \mathrm{~cm}^{2}$. In the pooled analysis, $79.7 \%$ in the dalbavancin group and $79.8 \%$ in the vancomycin-linezolid group met the primary endpoint of early clinical response with cessation of spread of infection-related erythema and absence of fever at 48 to $72 \mathrm{~h}$. Treatment success was also similar between groups in both studies when analyzed at the traditional endpoint and by infection type, underlying illness, infection severity, and pathogen [54].

\section{Safety and tolerability}

Across all studies, dalbavancin has been well tolerated with adverse events that were generally mild and similar to comparators [46,49,52-56]. The most commonly reported adverse events include nausea, diarrhea, fever, headache, oral candidiasis, and pruritis. Drug-related adverse events were rare and included one episode each of transient urticaria, mild forearm pain, cellulitis, anaphylactoid reaction, and mild leukopenia which resolved spontaneously [49,53,54]. DISCOVER 1 and DISCOVER 2 noted low rates of treatmentlimiting adverse effects in both arms: $2.1 \%$ for dalbavancin and $2.0 \%$ for vancomycinlinezolid [54]. There were no significant abnormalities in hematologic or clinical chemistry parameters, and there were no drug-related deaths across the studies.

Dalbavancin does not appear to have ototoxic effects, nor does it prolong the QTc interval [56,57]. A small study found no major change in the intestinal flora and no detectable Clostridium difficile among healthy patients who received a single $1000 \mathrm{mg}$ dose of dalbavancin [58]. There have not been any post-marketing adverse events reported to date.

\section{Regulatory affairs}

Dalbavancin was in development for about 15 years under four different companies [59]. Discovered by Marion Merrell Dow, it was first developed for clinical investigation by Biosearch Italia in 1999. Versicor Pharmaceuticals, Inc. partnered with Biosearch Italia and 
sought to begin US trials in 2000. In 2003, these companies merged to form Vicuron Pharmaceuticals, Inc., which conducted most of the early clinical trials. Pfizer Inc. acquired Vicuron in 2005. Vicuron and dalbavancin were acquired by Durata Therapeutics, Inc. in 2009, and Durata was acquired by Actavis in November, 2014.

From 2005 to 2007, the FDA issued three approvable action letters to Pfizer [59]. The first two involved manufacturing issues, and the third entailed evolving views of non-inferiority clinical trial design. Despite complete responses to all three, Pfizer withdrew the New Drug Application for dalbavancin in 2008. In its 2010 Guidance for Industry report on ABSSSI, the FDA recommended a primary efficacy endpoint based on early assessment of efficacy at 48 - 72 h. Durata's DISCOVER 1 and DISCOVER 2 trials were among the first ABSSSI studies registered using this new guidance [54].

Under the Generating Antibiotic Incentives Now (GAIN) title of the FDA Safety and Innovation Act, dalbavancin was designated as a Qualified Infectious Disease Product (QIDP) [60]. The QIDP designation granted dalbavancin priority review (6 months rather than 10 months) and provides additional marketing exclusivity to Durata Therapeutics/ Actavis, which currently markets dalbavancin. Dalbavancin was approved by the FDA in May 2014 and by the EMA in February 2015 for treatment of ABSSSI caused by susceptible Gram-positive bacteria in adults $\geq 18$ years of age $[15,16]$.

\section{Conclusion}

Dalbavancin is a recently approved, novel lipoglycopeptide which could be an important addition to the antimicrobial armamentarium. It has a well established activity against the Gram-positive organisms commonly involved in ABSSSI, including MRSA and other multidrug-resistant pathogens, and the $\mathrm{MIC}_{90}$ values for these organisms have remained stable over the past decade. Dalbavancin's high-protein binding and prolonged half-life allow for easily and consistently attainable therapeutic levels. Even with extensive protein binding, the free serum levels are adequate to provide excellent tissue penetration. Several clinical trials have demonstrated its tolerability, efficacy, and non-inferiority compared to standard therapy for ABSSSI. Dalbavancin's most unique feature is its once weekly dosing, and it has been approved as a $1000 \mathrm{mg}$ dose followed by $500 \mathrm{mg} 1$ week later (Table 2). This provides opportunities for increased adherence and fewer hospitalizations for complicated ABSSSI. Patients who once required hospitalization could reasonably be managed entirely as an outpatient, with one or both doses administered in the emergency department, clinic, or infusion center settings, or any combination thereof. Though convenient, dalbavancin's long half-life raises concerns about the management of adverse events, and close monitoring may be needed.

\section{Expert opinion}

Phase III trials found dalbavancin to be non-inferior to vancomycin when followed by linezolid and similar in efficacy to other conventional treatments for ABSSSI. From a drug development perspective, DISCOVER 1 [54] and DISCOVER 2 [54] are among the first trials to use early primary efficacy endpoints for the newly defined indication ABSSSI. 
Dalbavancin was also among the first drugs to earn a QIDP designation under the GAIN Act, with expedited review and marketing incentives for Durata/Actavis. As such, dalbavancin may not only be a novel treatment option for ABSSSI, but also an important example to foster future drug development efforts.

Current evaluation of a single dose study of dalbavancin for ABSSSI, a planned Phase III trial for community-acquired pneumonia, and trials in pediatric populations will further define its therapeutic use [61]. Additional studies are needed to determine its role in bacterial bone and joint infections, hospital-acquired pneumonia, endocarditis, deep-tissue abscess, and central nervous system infections. Cost-effectiveness analyses may support the use of dalbavancin over less expensive options as it may enable outpatient treatment in patients who formerly would have been admitted to the hospital. Post-marketing surveillance will also be important for a drug with such a prolonged half-life as managing adverse events may be challenging. Additionally, direct comparison with the novel lipoglycopeptide oritavancin in clinical trials would be of interest given the shared spectrum of activity and long half-life.

The best use of dalbavancin from an antimicrobial steward-ship standpoint remains unclear. The empiric use of a drug with an 8- to 10-day half-life may not be appropriate if therapy can be tailored after $2-3$ days based on microbiologic culture data. The inability to deescalate may expose patients to prolonged selective pressure. It would be reasonable to reserve empirical dalbavancin for ABSSSI cases that are not amenable to culture in settings with high MRSA rates.

Dalbavancin presents an attractive alternative for patients who need prolonged intravenous antimicrobial therapy. Once weekly dosing obviates the need for an indwelling intravenous catheter and its associated risks, including infection and venous thromboembolism. Dalbavancin also provides an option for injection drug users who may be at high risk for inappropriate use of an intravenous catheter. This treatment modality is also more convenient for patients and could reduce costs associated with long courses of intravenous antibiotics, including home nursing care, admission to skilled nursing facilities, and hospitalization for related complications. It may also prove useful for patients who may not be adherent to complicated oral regimens (e.g., TMP/SMX plus cephalexin) and for those in whom conventional treatments are limited by allergy or drug toxicity.

After a long development process, dalbavancin was approved in the US in 2014 and in Europe in 2015, and stands to become a valuable therapeutic option for ABSSSI.

Dalbavancin has excellent activity against bacteria most commonly implicated in ABSSSI, including multi-drug-resistant strains of $S$. aureus, and has not had any evidence of resistance to date. Reported adverse events are mild and no significant drug interactions have been noted to date. In the current era of increasing infections caused by multi-drugresistant pathogens, dalbavancin is a safe and efficacious option for patients with ABSSSI. Future studies will hopefully define its role in other infections, including osteomyelitis and bloodstream infection. 


\section{Acknowledgments}

SK Ramdeen is supported by NIH Training Grant number 5T32 AIO55412-09. H Boucher serves or has served as a consultant to Merck, the National Institutes of Health (adjudication committee) and Theravance.

\section{Bibliography}

Papers of special note have been highlighted as either of interest $(\bullet)$ or of considerable interest $(\bullet)$ to readers.

1. Food and Drug Administration. Guidance for industry acute bacterial skin and skin structure infections: developing drugs for treatment. 2013. Available from: http://www.fda.gov/downloads/ Drugs/Guidances/ucm071185.pdf

2. Edelsberg J, Taneja C, Zervos M, et al. Trends in US hospital admissions for skin and soft tissue infections. Emerg Infect Dis. 2009; 15(9):1516-18. [PubMed: 19788830]

3. Hersh AL, Chambers HF, Maselli JH, Gonzales R. National trends in ambulatory visits and antibiotic prescribing for skin and soft-tissue infections. Arch Intern Med. 2008; 168(14):1585-91. [PubMed: 18663172]

4. May L, Mullins P, Pines J. Demographic and treatment patterns for infections in ambulatory settings in the United States, 2006-2010. Acad Emerg Med. 2014; 21(1):17-24. [PubMed: 24552520]

5. Wilder JR, Wegener DT, David MZ, et al. A national survey of skin infections, care behaviors and MRSA knowledge in the United States. PLoS ONE. 2014; 9(8):e104277. [PubMed: 25137061]

6. Dukic VM, Lauderdale DS, Wilder J, et al. Epidemics of community-associated methicillin-resistant Staphylococcus aureus in the United States: A meta-analysis. PLoS ONE. 2013; 8(1):e52722. [PubMed: 23300988]

7. Suaya JA, Mera RA, Cassidy A, et al. Incidence and cost of hospitalizations associated with Staphylococcus aureus skin and soft tissue infections in the United States from 2001 through 2009. BMC Infect Dis. 2014; 14:296-303. [PubMed: 24889406]

8. Ray GT, Suaya JA, Baxter R. Incidence, microbiology, and patient characteristics of skin and softtissue infections in a U.S. population: A retrospective population-based study. BMC Infect Dis. 2013; 13:252. [PubMed: 23721377]

9. Johnson AP. Methicillin-resistant Staphylococcus aureus: The European landscape. J Antimicrob Chemother. 2011; 66(Suppl 4):iv43-8. [PubMed: 21521706]

10. Dryden M, Andrasevic AT, Bassetti M, et al. A European survey of antibiotic management of methicillin-resistant Staphylococcus aureus infection: current clinical opinion and practice. Clin Microbiol Infect. 2010; 16(Suppl 1):3-30. [PubMed: 20222890]

11. Stevens DL, Bisno AL, Chambers HF, et al. Practice guidelines for the diagnosis and management of skin and soft tissue infections: 2014 update by the infectious diseases society of America. Clin Infect Dis. 2014; 59(2):e10-52. [PubMed: 24973422]

12. Nannini E, Murray BE, Arias CA. Resistance or decreased susceptibility to glycopeptides, daptomycin, and linezolid in methicillin-resistant Staphylococcus aureus. Curr Opin Pharmacol. 2010; 10(5):516-21. [PubMed: 20598637]

13. Long SW, Olsen RJ, Mehta SC, et al. PBP2a mutations causing high-level ceftaroline resistance in clinical methicillin-resistant Staphylococcus aureus isolates. Antimicrob Agents Chemother. 2014; 58(11):6668-74. [PubMed: 25155594]

14. Jenkins TC, Sabel AL, Sarcone EE, et al. Skin and soft-tissue infections requiring hospitalization at an academic medical center: Opportunities for antimicrobial stewardship. Clin Infect Dis. 2010; 51(8):895-903. [PubMed: 20839951]

15. Food and Drug Administration. [Last accessed 21 March 2015] FDA approves dalvance to treat skin infections [Internet]. 2014. Available from: http://www.fda.gov/newsevents/newsroom/ pressannouncements/ucm398724.htm

16. European Medicines Agency. [Last accessed 17 April 2015] Xydalba authorisation details [Internet]. 2015. Available from: http://www.ema.europa.eu/ema/index.jsp?curl=pages/medicines/ human/medicines/002840/human_med_001848.jsp\&mid=WC0b01ac058001d124 
17. Corey GR, Kabler H, Mehra P, et al. Single-dose oritavancin in the treatment of acute bacterial skin infections. N Engl J Med. 2014; 370(23):2180-90. [PubMed: 24897083]

18. Moran GJ, Fang E, Corey GR, et al. Tedizolid for 6 days versus linezolid for 10 days for acute bacterial skin and skin-structure infections (ESTABLISH-2): A randomised, double-blind, phase 3, non-inferiority trial. Lancet Infect Dis. 2014; 14(8):696-705. [PubMed: 24909499]

19. Deresinski S. Counterpoint: Vancomycin and Staphylococcus aureus-an antibiotic enters obsolescence. Clin Infect Dis. 2007; 44(12):1543-8. [PubMed: 17516396]

20. Bassetti M, Merelli M, Temperoni C, Astilean A. New antibiotics for bad bugs: Where are we? Ann Clin Microbiol Antimicrob. 2013; 12:22. [PubMed: 23984642]

21. Pew Charitable Trusts. [Last accessed 6 April 2015] Antibiotics currently in clinical development [Internet]. 2014. Available from: http://www.pewtrusts.org/en/multimedia/data-visualizations/ 2014/antibiotics-currently-in-clinical-development

22. Malabarba A, Goldstein BP. Origin, structure, and activity in vitro and in vivo of dalbavancin. $\mathrm{J}$ Antimicrob Chemother. 2005; 55(Suppl 2):ii15-20. [PubMed: 15750032]

23. Economou NJ, Nahoum V, Weeks SD, et al. A carrier protein strategy yields the structure of dalbavancin. J Am Chem Soc. 2012; 134(10):4637-45. [PubMed: 22352468] Defining dalbavancin's crystal structure and how it may relate to binding its target site.

24. Bowker KE, Noel AR, MacGowan AP. Pharmacodynamics of dalbavancin studied in an in vitro pharmacokinetic system. J Antimicrob Chemother. 2006; 58(4):802-5. [PubMed: 16891629]

25. Lin G, Credito K, Ednie LM, Appelbaum PC. Antistaphylococcal activity of dalbavancin, an experimental glycopeptide. Antimicrob Agents Chemother. 2005; 49(2):770-2. [PubMed: 15673763]

26. Dowell JA, Goldstein BP, Buckwalter M, et al. Pharmacokinetic-pharmacodynamic modeling of dalbavancin, a novel glycopeptide antibiotic. J Clin Pharmacol. 2008; 48(9):1063-8. [PubMed: 18633123]

27. Jabes D, Candiani G, Romano G, et al. Efficacy of dalbavancin against methicillin-resistant Staphylococcus aureus in the rat granuloma pouch infection model. Antimicrob Agents Chemother. 2004; 48(4):1118-23. [PubMed: 15047510]

28. Andes D, Craig WA. In vivo pharmacodynamic activity of the glycopeptide dalbavancin. Antimicrob Agents Chemother. 2007; 51(5):1633-42. [PubMed: 17307987]

29. Streit JM, Fritsche TR, Sader HS, Jones RN. Worldwide assessment of dalbavancin activity and spectrum against over 6,000 clinical isolates. Diagn Microbiol Infect Dis. 2004; 48(2):137-43. [PubMed: 14972384]

30. Streit JM, Sader HS, Fritsche TR, Jones RN. Dalbavancin activity against selected populations of antimicrobial-resistant Gram-positive pathogens. Diagn Microbiol Infect Dis. 2005; 53(4):307-10. [PubMed: 15922534]

31. Gales AC, Sader HS, Jones RN. Antimicrobial activity of dalbavancin tested against Gram-positive clinical isolates from Latin American medical centres. Clin Microbiol Infect. 2005; 11(2):95-100. [PubMed: 15679482]

32. Biedenbach DJ, Ross JE, Fritsche TR, et al. Activity of dalbavancin tested against Staphylococcus spp. and beta-hemolytic Streptococcus spp. isolated from 52 geographically diverse medical centers in the United States. J Clin Microbiol. 2007; 45(3):998-1004. [PubMed: 17215346]

33. Biedenbach DJ, Jones RN. Multicenter evaluation of the in vitro activity of dalbavancin tested against Staphylococci and Streptococci in 5 European countries: Results from the DECIDE surveillance program (2007). Diagn Microbiol Infect Dis. 2009; 64(2):177-84. [PubMed: 19249179]

34. Goldstein EJ, Citron DM, Warren YA, et al. In vitro activities of dalbavancin and 12 other agents against 329 aerobic and anaerobic Gram-positive isolates recovered from diabetic foot infections. Antimicrob Agents Chemother. 2006; 50(8):2875-9. [PubMed: 16870792]

35. Huang V, Cheung CM, Kaatz GW, Rybak MJ. Evaluation of dalbavancin, tigecycline, minocycline, tetracycline, teicoplanin and vancomycin against community-associated and multidrug-resistant hospital-associated meticillin-resistant Staphylococcus aureus. Int J Antimicrob Agents. 2010; 35(1):25-9. [PubMed: 19900792] 
36. Lopez S, Hackbarth C, Romano G, et al. In vitro antistaphylococcal activity of dalbavancin, a novel glycopeptide. J Antimicrob Chemother. 2005; 55(Suppl 2):ii21-4. [PubMed: 15750033]

37. Goldstein BP, Draghi DC, Sheehan DJ, et al. Bactericidal activity and resistance development profiling of dalbavancin. Antimicrob Agents Chemother. 2007; 51(4):1150-4. [PubMed: 17220411]

38. Jones RN, Stilwell MG, Sader HS, et al. Spectrum and potency of dalbavancin tested against 3322 Gram-positive cocci isolated in the United States surveillance program (2004). Diagn Microbiol Infect Dis. 2006; 54(2):149-53. [PubMed: 16426793]

39. Biedenbach DJ, Bell JM, Sader HS, et al. Activities of dalbavancin against a worldwide collection of 81,673 gram-positive bacterial isolates. Antimicrob Agents Chemother. 2009; 53(3):1260-3. [PubMed: 19124664]

40. Jones RN, Sader HS, Flamm RK. Update of dalbavancin spectrum and potency in the USA: Report from the SENTRY antimicrobial surveillance program (2011). Diagn Microbiol Infect Dis. 2013; 75(3):304-7. [PubMed: 23357293]

41 • Jones RN, Flamm RK, Sader HS. Surveillance of dalbavancin potency and spectrum in the United States (2012). Diagn Microbiol Infect Dis. 2013; 76(1):122-3. [PubMed: 23433533] Most recent surveillance study demonstrating maintenance of dalbavancin's potency and spectrum of activity.

42. Goldstein EJ, Citron DM, Merriam CV, et al. In vitro activities of dalbavancin and nine comparator agents against anaerobic Gram-positive species and Corynebacteria. Antimicrob Agents Chemother. 2003; 47(6):1968-71. [PubMed: 12760876]

43. Jones RN, Stilwell MG. Comprehensive update of dalbavancin activity when tested against uncommonly isolated Streptococci, Corynebacterium spp., Listeria monocytogenes, and Micrococcus spp. (1357 strains). Diagn Microbiol Infect Dis. 2013; 76(2):239-40. [PubMed: 23414746]

44. Johnson DM, Fritsche TR, Sader HS, Jones RN. Evaluation of dalbavancin in combination with nine antimicrobial agents to detect enhanced or antagonistic interactions. Int J Antimicrob Agents. 2006; 27(6):557-60. [PubMed: 16698238]

45. Cavaleri M, Riva S, Valagussa A, et al. Pharmacokinetics and excretion of dalbavancin in the rat. J Antimicrob Chemother. 2005; 55(Suppl 2):ii31-5. [PubMed: 15750035]

46•. Leighton A, Gottlieb AB, Dorr MB, et al. Tolerability, pharmacokinetics, and serum bactericidal activity of intravenous dalbavancin in healthy volunteers. Antimicrob Agents Chemother. 2004; 48(3):940-5. [PubMed: 14982787] Phase I study supporting once-weekly dosing.

47•. Dorr MB, Jabes D, Cavaleri M, et al. Human pharmacokinetics and rationale for once-weekly dosing of dalbavancin, a semi-synthetic glycopeptide. J Antimicrob Chemother. 2005; 55(Suppl 2):ii25-30. [PubMed: 15750034] Human PK study supporting once-weekly dosing.

48. Nicolau DP, Sun HK, Seltzer E, et al. Pharmacokinetics of dalbavancin in plasma and skin blister fluid. J Antimicrob Chemother. 2007; 60(3):681-4. [PubMed: 17631507]

49. Dunne MW, Puttagunta S, Sprenger CR, et al. Extended-duration dosing and distribution of dalbavancin into bone and articular tissue. Antimicrob Agents Chemother. 2015; 59(4):1849-55. [PubMed: 25561338]

50. Buckwalter M, Dowell JA. Population pharmacokinetic analysis of dalbavancin, a novel lipoglycopeptide. J Clin Pharmacol. 2005; 45(11):1279-87. [PubMed: 16239361]

51. Marbury T, Dowell JA, Seltzer E, Buckwalter M. Pharmacokinetics of dalbavancin in patients with renal or hepatic impairment. J Clin Pharmacol. 2009; 49(4):465-76. [PubMed: 19318696]

52••. Seltzer E, Dorr MB, Goldstein BP, et al. Once-weekly dalbavancin versus standard-of-care antimicrobial regimens for treatment of skin and soft-tissueinfections. Clin Infect Dis. 2003; 37(10):1298-303. [PubMed: 14583862] Phase II study demonstrating the efficacy of dalbavancin in ABSSSI compared to conventional therapy.

53••. Jauregui LE, Babazadeh S, Seltzer E, et al. Randomized, double-blind comparison of onceweekly dalbavancin versus twice-daily linezolid therapy for the treatment of complicated skin and skin structure infections. Clin Infect Dis. 2005; 41(10):1407-15. [PubMed: 16231250] Phase III study which found that dalbavancin is comparable to linezolid in tolerability and efficacy.

54••. Boucher HW, Wilcox M, Talbot GH, et al. Once-weekly dalbavancin versus daily conventional therapy for skin infection. N Engl J Med. 2014; 370(23):2169-79. [PubMed: 24897082] 
DISCOVER-1 and DISCOVER-2, pivotal Phase III trials demonstrating dalbavancin's noninferiority versus vancomycin that led to its approval in the US and Europe.

55. Raad I, Darouiche R, Vazquez J, et al. Efficacy and safety of weekly dalbavancin therapy for catheter-related bloodstream infection caused by Gram-positive pathogens. Clin Infect Dis. 2005; 40(3):374-80. [PubMed: 15668859]

56. Campbell K, Kelly E, Targovnik N, et al. Audiologic monitoring for potential ototoxicity in a phase I clinical trial of a new glycopeptide. J Am Acad Audiol. 2003; 14(3):157-68. [PubMed: 12859140]

57. Dunne MW, Zhou M, Darpo B. A thorough QT study with dalbavancin: A novel lipoglycopeptide antibiotic for the treatment of acute bacterial skin and skin-structure infections. Int J Antimicrob Agents. 2015; 45(4):393-8. [PubMed: 25681068]

58. Nord CE, Rasmanis G, Wahlund E. Effect of dalbavancin on the normal intestinal microflora. J Antimicrob Chemother. 2006; 58(3):627-31. [PubMed: 16840427]

59. Durata Therapeutics International B.V. Dalbavancin for injection for treatment of acute bacterial skin and skin structure infections. Briefing Document. FDA Anti-Infective Drugs Advisory Committee; 2014. Report No: ND 021-883

60. United States Food and Drug Administration Center for Drug Evaluation and Research. Novel new drugs 2014 summary. 2015

61. National Institutes of Health. [Last accessed 17 April 2015] Search results for dalbavancin [Internet]. 2015. Available from: https://clinicaltrials.gov/ct2/results?intr=\%22Dalbavancin $\% 22$ 


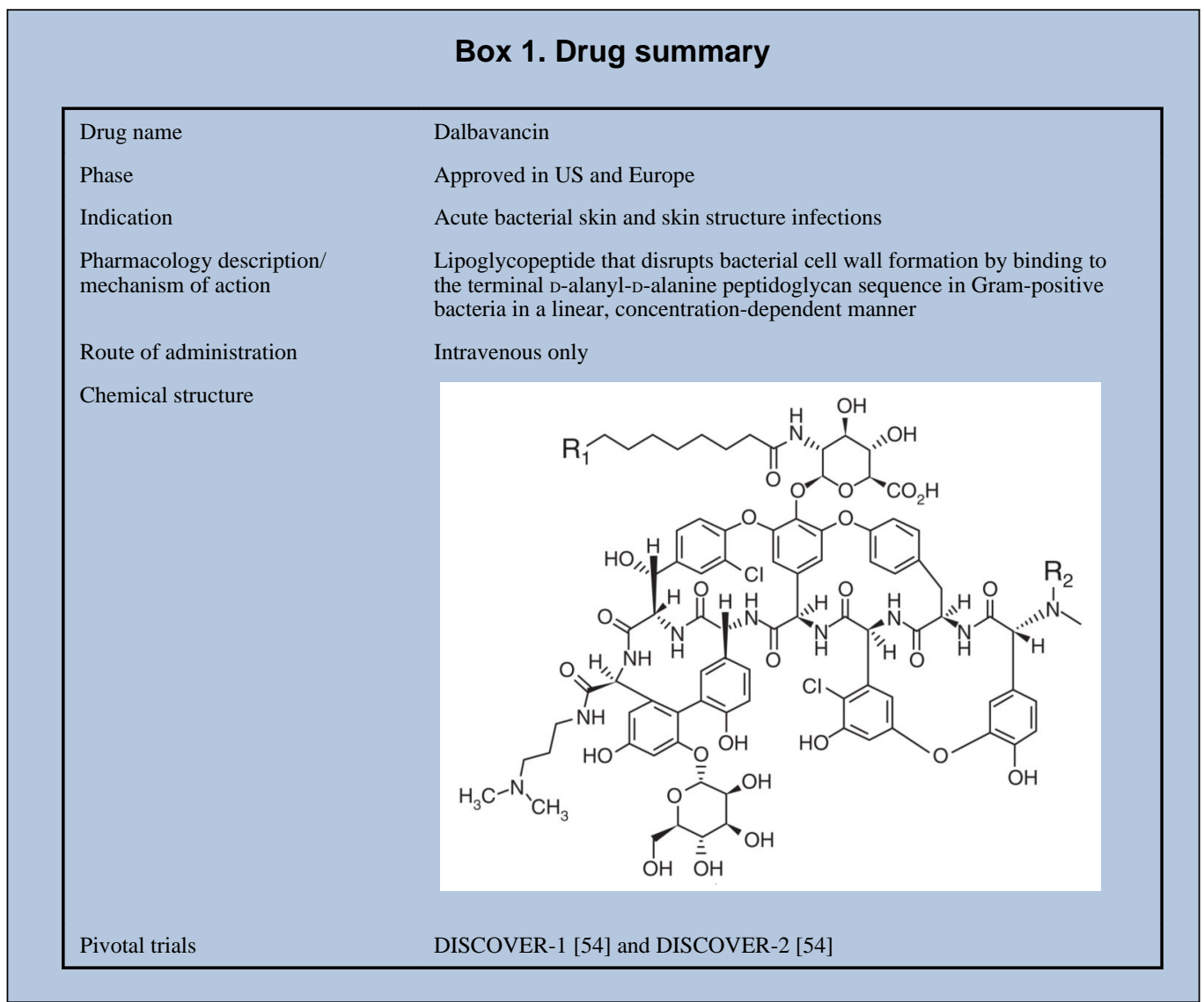


Table 1

Summary of DISCOVER 1 and DISCOVER 2 studies

\begin{tabular}{|c|c|c|c|c|c|c|}
\hline \multirow[t]{3}{*}{ Characteristic } & \multicolumn{2}{|c|}{ DISCOVER 1} & \multicolumn{2}{|c|}{ DISCOVER 2} & \multicolumn{2}{|c|}{ DISCOVER studies } \\
\hline & Dalbavancin & $\begin{array}{l}\text { Vancomycin/ } \\
\text { linezolid }\end{array}$ & Dalbavancin & $\begin{array}{l}\text { Vancomycin/ } \\
\text { linezolid }\end{array}$ & Dalbavancin & $\begin{array}{l}\text { Vancomycin/ } \\
\text { linezolid }\end{array}$ \\
\hline & $\mathrm{N}=\mathbf{2 8 8}$ & $\mathrm{N}=\mathbf{2 8 5}$ & $\mathbf{N}=\mathbf{3 7 1}$ & $N=368$ & $N=659$ & $N=653$ \\
\hline \multicolumn{7}{|l|}{ Age - years } \\
\hline Mean & 48.8 & 48.9 & 49.1 & 51.4 & 48.9 & 50.3 \\
\hline Range & 18,84 & 18,84 & 18,85 & 18,84 & 18,85 & 18,84 \\
\hline Male sex n, $(\%)$ & $170(59.0)$ & $173(60.7)$ & $223(60.1)$ & $201(54.6)$ & $393(59.6)$ & $374(57.3)$ \\
\hline Diabetes mellitus n, (\%) & $43(14.9)$ & $30(10.5)$ & $35(9.4)$ & $62(16.8)$ & $78(11.8)$ & $92(14.1)$ \\
\hline SIRS n, $(\%)^{*}$ & $175(61.6)$ & $175(61.6)$ & $157(42.7)$ & $161(43.8)$ & $332(50.9)$ & $336(51.5)$ \\
\hline \multicolumn{7}{|l|}{ Infection type $n,(\%)$} \\
\hline Major abscess & $72(25.0)$ & $86(30.2)$ & $90(24.3)$ & 87 (23.6) & $162(24.6)$ & $173(26.5)$ \\
\hline Cellulitis & $156(54.2)$ & $147(51.6)$ & $198(53.4)$ & $202(54.9)$ & $354(53.7)$ & $349(53.4)$ \\
\hline Wound/surgical site infection & $60(20.8)$ & $52(18.2)$ & $82(22.1)$ & $79(21.5)$ & $142(21.5)$ & $131(20.1)$ \\
\hline $\begin{array}{l}\text { Infection area, median } \mathrm{cm}^{2} \text {, } \\
\text { (range })^{\frac{t^{*}}{}}\end{array}$ & $\begin{array}{l}333.0 \\
(26,3400)\end{array}$ & $367.8(78,3675)$ & $313.50(85,5100)$ & $362.40(72,3922)$ & $324.0(26,5100)$ & $366.8(72,3922)$ \\
\hline $\begin{array}{l}\text { Patients with a pathogen } \\
\text { isolated } \\
\text { at baseline, } \mathrm{n}\end{array}$ & 153 & 155 & 184 & 174 & 337 & 329 \\
\hline MRSA, n & 44 & 39 & 46 & 28 & 90 & 67 \\
\hline MSSA, n & 78 & 88 & 89 & 101 & 167 & 189 \\
\hline S. pyogenes, $\mathrm{n}$ & 12 & 14 & 25 & 22 & 37 & 36 \\
\hline \multicolumn{7}{|c|}{ Primary endpoint success rates at $48-72 \mathrm{~h}$ (early clinical response, ITT population) } \\
\hline Dalbavancin $\mathrm{n} / \mathrm{N}(\%)$ & \multicolumn{2}{|c|}{$240 / 288(83.3)$} & \multicolumn{2}{|c|}{ 285/371 (76.8) } & \multicolumn{2}{|c|}{$525 / 659$ (79.7) } \\
\hline Vancomycin/linezolid n/N(\%) & \multicolumn{2}{|c|}{$233 / 285(81.8)$} & \multicolumn{2}{|c|}{$288 / 368(78.3)$} & \multicolumn{2}{|c|}{$521 / 653(79.8)$} \\
\hline $\begin{array}{l}\text { Absolute difference in } \\
\text { success rates } \\
(95 \% \mathrm{CI})\end{array}$ & \multicolumn{2}{|c|}{$1.5(-4.6,7.9)$} & \multicolumn{2}{|c|}{$-1.5(-7.4,4.6)$} & \multicolumn{2}{|c|}{$-0.1(-4.5,4.2)$} \\
\hline
\end{tabular}

Data from [54].

MRSA: Methicillin-resistant Staphylococcus aureus.

* SIRS, systemic inflammatory response syndrome, is defined as having two or more of the following: temperature $\left\langle 36^{\circ} \mathrm{C}\right.$ or $>38^{\circ} \mathrm{C}$; heart rate $>$ 90 beats per minute; respiratory rate $>20$ breaths per minute; $\mathrm{WBC}$ count $<4000$ cells $/ \mathrm{mm}^{3}$ or $>12,000$ cells $/ \mathrm{mm}^{3}$ or $>10 \%$ band forms.

$¥$ Area of erythema defined as longest length $\times$ widest width perpendicular to length 
Table 2

Dalbavancin dosing regimen, dose adjustment, length of therapy, and cost

\begin{tabular}{ll}
\hline Dosing & $\begin{array}{c}1000 \mathrm{mg} \text { followed } 1 \text { week later by } 500 \mathrm{mg} \\
\text { For creatinine clearance less than } 30 \mathrm{ml} / \mathrm{min} \text { and not on hemodialysis: } 750 \mathrm{mg} \\
\text { followed one week later by } 375 \mathrm{mg} \\
\text { For hepatic impairment: no dose adjustment needed }\end{array}$ \\
Two dose regimen over 2 weeks \\
Wholesale acquisition cost & US\$1490/500 mg vial (as of 3/2/15) \\
\hline
\end{tabular}

